\title{
Association of Red Blood Cell Distribution Width Levels with Severity of Coronary Artery Disease in Patients with Non-ST Elevation Myocardial Infarction
}

\author{
Omer Sahin ${ }^{\mathrm{a}}$ Mahmut Akpek ${ }^{\mathrm{a}}$ Bahadir Sarli ${ }^{\mathrm{a}} \quad$ Ahmet Oguz Baktir ${ }^{\mathrm{a}}$ \\ Goktug Savas $^{b}$ Serhat Karadavut ${ }^{b}$ Deniz Elcik ${ }^{b}$ Hayrettin Saglam ${ }^{a}$ \\ Mehmet Gungor Kaya ${ }^{b}$ Huseyin Arinc ${ }^{a}$ \\ ${ }^{a}$ Department of Cardiology, Kayseri Education and Research Hospital, and ${ }^{b}$ Department of Cardiology, \\ Erciyes University School of Medicine, Kayseri, Turkey
}

\section{Key Words}

Red blood cell distribution width - Non-ST elevation myocardial infarction - SYNTAX score - Coronary artery disease

\begin{abstract}
Objectives: The aim of this study was to evaluate the association of the levels of red blood cell distribution width (RDW) with the severity of atherosclerosis and to determine whether or not the RDW level on admission is an independent predictor of all-cause mortality in patients with non-ST elevation myocardial infarction (NSTEMI). Materials and Methods: A total of 335 consecutive patients with NSTEMI were enrolled in this study. The patients were divided into high $(n=105)$ and low $(n=230)$ SYNTAX groups. The high SYNTAX group was defined as patients with a value in the third tertile (SYNTAX score, SXscore $\geq 12$ ), while the low SYNTAX group was defined as those with a value in the lower 2 tertiles (SXscore $<12)$. The high RDW group $(n=152)$ was defined as patients with RDW $>14.25 \%$ and the low RDW group ( $n=183$ ) as those with RDW $\leq 14.25 \%$. All-cause mortality was followed up to 38 months. Results: The mean follow-up period was $18 \pm 11$ months. The RDW levels of patients were significantly higher in the high SYNTAX group
\end{abstract}

than in the low SYNTAX group $(15.2 \pm 1.8$ vs. $14.2 \pm 1.2, p<$ $0.001)$. Pearson's coefficients were used to determine the degree of association between RDW levels and SXscore and also between RDW levels and high-sensitivity C-reactive protein. There was a significant correlation between RDW levels and SXscore $(r=0.460, p<0.001)$. Also, there was a significant correlation between RDW levels and high-sensitivity Creactive protein $(r=0.180, p=0.001)$. All-cause mortality rate was not significantly different between the high and low RDW groups (log-rank, $p=0.621$ ). Conclusion: RDW levels were independently associated with high SXscore but were not associated with long-term mortality in NSTEMI patients.

(C) 2014 S. Karger AG, Basel

\section{Introduction}

Red blood cell distribution width (RDW) is a numerical value measure of the variability in size of circulating erythrocytes. High RDW values show greater variation in size than recent studies have shown, indicating that high RDW values are an independent predictor of prognosis in patients with cardiovascular diseases such as acute myocardial infarction $[1,2]$ and non-ST elevation myocardial infarction (NSTEMI) [3]. Azab et al. [3] showed

\begin{tabular}{ll}
\hline KARGER 125:s & $\begin{array}{l}\text { ( ) 2014 S. Karger AG, Basel } \\
1011-7571 / 14 / 0242-0178 \$ 39.50 / 0 \quad \text { Karger }\end{array}$ \\
$\begin{array}{l}\text { E-Mail karger@karger.com } \\
\text { www.karger.com/mpp }\end{array}$ & $\begin{array}{l}\text { Thisis an Open Access article licensed under the terms of the } \\
\text { Creative Commons Attribution-NonCommercial 3.0 Un- } \\
\text { ported license (CC BY-NC) (www.karger.com/OA-license), } \\
\text { applicable to the online version of the article only. Distribu- } \\
\text { tion permitted for non-commercial purposes only. }\end{array}$
\end{tabular}

Omer Sahin, MD

Department of Cardiology

Kayseri Education and Research Hospital

TR-38039 Kayseri (Turkey)

E-Mail dr.osahin@yahoo.com 
in their study that RDW is an independent predictor of all-cause long-term mortality in NSTEMI patients. It has also been reported that high levels of RDW are associated with the presence, severity and complexity of coronary artery disease in patients with stable angina pectoris and ST elevation myocardial infarction (STEMI) $[4,5]$.

To the best of our knowledge, the association between RDW levels and the severity of coronary artery disease assessed by the SYNTAX score (SXscore) in patients with NSTEMI has not been reported. Therefore, the aim of this study was to evaluate the association between RDW levels and the severity of atherosclerosis and to determine whether or not the RDW level on admission is an independent predictor of all-cause mortality in patients with NSTEMI.

\section{Subjects and Methods}

Patients with NSTEMI admitted to the Emergency Department from March 2010 to November 2012 were included in the study. NSTEMI was defined as follows: typical chest pain, a positive troponin-I level (defined in our clinical laboratory as $>0.02 \mathrm{ng} / \mathrm{ml}$ ) and no evidence of ST segment elevation on 12-lead electrocardiogram. Exclusion criteria were a history of any coronary artery disease shown on coronary angiogram, cardiogenic shock, heart failure, renal disease (or serum creatinine level $>1.5 \mathrm{mg} / \mathrm{dl}$ ), anemia, clinical evidence of active infection, active cancer, hematological proliferative diseases, active or chronic inflammatory or autoimmune diseases, pregnancy, recent blood transfusion, and severe arrhythmia, in the light of previous studies [6,7].

A total of 449 eligible consecutive patients were evaluated. Of these, the following were excluded: patients who refused to give informed consent $(n=57)$; patients with missing or unavailable blood sample parameters for RDW and high-sensitivity C-reactive protein $($ hs-CRP) values $(\mathrm{n}=23)$; patients with normal coronary arteries after the administration of intracoronary nitrate during the diagnostic coronary angiogram $(n=17)$; patients with pericarditis $(n=11)$, and patients with myocarditis $(n=6)$. Hence, 335 patients with NSTEMI were enrolled in this study.

Selective coronary angiography was performed in multiple orthogonal views using standard techniques. Each coronary lesion producing a $\geq 50 \%$ luminal obstruction in vessels $\geq 1.5 \mathrm{~mm}$ was separately scored and added to provide the vessel SXscore. The SXscore was calculated using dedicated software that integrates the number of lesions with their specific weighting factors, based on the amount of myocardium distal to the lesion according to the score of Leaman et al. [8], and the morphological features of each single lesion, as previously reported [9]. Diagnostic angiograms for all NSTEMI patients were reviewed by 2 researchers blinded to the clinical data for the determination of the culprit artery.

The study population was divided into tertiles according to the SXscore. The high SYNTAX group $(\mathrm{n}=105)$ was defined as patients with a value in the third tertile (SXscore $\geq 12$ ) and the low SYNTAX group $(n=230)$ as those with a value in the lower 2 tertiles (SXscore $<12$ ). According to receiver-operating characteristic (ROC) analysis, the patients were divided into two groups; the high RDW group was defined as patients with RDW $>14.25 \%(n=152)$ and the low RDW group as those with RDW $\leq 14.25 \%(\mathrm{n}=183)$, according to a previous study [10]. The mortality data were obtained from the hospital records or by interviewing (directly or by telephone) the patients, families or personal physicians.

In all patients, antecubital venous blood samples for laboratory analysis were drawn on admission to the Emergency Department. Glucose, creatinine, peak cardiac-MB and lipid profiles were analyzed using an autoanalyzer (Roche Diagnostic Modular Systems, Tokyo, Japan). hs-CRP was measured using a BN2 model nephelometer (Dade-Behring, Schwalbach, Germany) within 5 min of sampling. Common blood count parameters were measured using a Sysmex K-1000 autoanalyzer within 5 min of sampling.

Transthoracic echocardiography was performed for each patient immediately in the Cardiac Intensive Care Unit. All measurements were performed using a commercially available machine (Vivid 7 GE Medical System, Horten, Norway) with a $3.5-\mathrm{MHz}$ transducer. The Simpson method in the 2-dimensional echocardiographic apical 4-chamber view was used to assess left ventricular ejection fraction (LVEF).

Continuous variables were tested for normal distribution by the Kolmogorov-Smirnov test. Continuous data are reported as means and standard deviations or medians. Continuous variables were compared using Student's t test or the Mann-Whitney U test between groups. Categorical variables were summarized as percentages and compared with the $\chi^{2}$ test. Pearson's coefficients were used to determine the degree of association between RDW levels and SXscore and also between hs-CRP and RDW levels. A twosided $p$ value $<0.05$ was considered significant. ROC analysis was used to demonstrate the sensitivity and specificity of RDW and the optimal cut-off value for predicting a high SXscore in patients with NSTEMI. The effects of different variables on high levels of SXscore were calculated using univariate analysis for each. The variables for which the nonadjusted $\mathrm{p}$ value was $<0.10$ in linear regression analysis were identified as potential risk markers and included in the full model. Backward elimination multivariate linear regression analysis was used to reduce the model, and potential risk markers were eliminated using likelihood ratio tests. The cumulative survival curve to all-cause mortality for RDW was analyzed by using the Kaplan-Meier method, with differences assessed by log-rank tests. Survival analyses were performed using multivariate Cox regression models to identify independent predictors of all-cause mortality. The following variables were incorporated in the model: age, diabetes mellitus, hypertension, treatment option, LVEF, SYNTAX group, RDW, troponin-I levels, creatine kinase-MB, low-density lipoprotein cholesterol (LDL-C), and hsCRP. The variables for which the unadjusted $p$ value was $<0.10$ in univariate Cox regression analysis were identified as potential risk markers and included in the full model. A p value $<0.05$ was considered statistically significant, and the confidence interval (CI) was $95 \%$. All statistical analyses were performed with SPSS version 15 (SPSS, Inc., Chicago, Ill., USA).

\section{Results}

Baseline characteristics are shown in table 1 . The mean age was $63 \pm 13$ years in the low SYNTAX group and 66 \pm 10 years in the high SYNTAX group; the difference was 
Table 1. Baseline characteristics of patients

\begin{tabular}{|c|c|c|c|}
\hline & $\begin{array}{l}\text { Low SYNTAX } \\
\text { group }(n=230)\end{array}$ & $\begin{array}{l}\text { High SYNTAX } \\
\text { group }(\mathrm{n}=105)\end{array}$ & $\begin{array}{l}\mathrm{p} \\
\text { value }\end{array}$ \\
\hline Age, years & $63 \pm 13$ & $66 \pm 10$ & 0.009 \\
\hline Sex, male & $147(64 \%)$ & $74(71 \%)$ & 0.240 \\
\hline Hypertension & $113(49 \%)$ & $57(54 \%)$ & 0.381 \\
\hline Diabetes mellitus & $64(28 \%)$ & $38(36 \%)$ & 0.123 \\
\hline Current smoker & $77(33 \%)$ & $39(37 \%)$ & 0.513 \\
\hline $\mathrm{SBP}, \mathrm{mm} \mathrm{Hg}$ & $132 \pm 17$ & $133 \pm 17$ & 0.731 \\
\hline DBP, mm Hg & $86 \pm 10$ & $85 \pm 11$ & 0.839 \\
\hline BMI & $27 \pm 3$ & $26 \pm 3$ & 0.950 \\
\hline LVEF on admission (\%) & $54 \pm 9$ & $51 \pm 9$ & 0.004 \\
\hline \multicolumn{4}{|l|}{ Acute treatment } \\
\hline PCI & $202(88 \%)$ & $66(63 \%)$ & $<0.001$ \\
\hline CABG surgery & $7(3 \%)$ & $30(29 \%)$ & $<0.001$ \\
\hline Medical & $21(9 \%)$ & $9(9 \%)$ & $<0.001$ \\
\hline Left anterior descending & $106(46 \%)$ & $82(78 \%)$ & $<0.001$ \\
\hline Right & $140(61 \%)$ & $56(53 \%)$ & 0.194 \\
\hline Circumflex & $51(22 \%)$ & $47(45 \%)$ & $<0.001$ \\
\hline Left main coronary artery & $0(0 \%)$ & $3(0.3 \%)$ & 0.010 \\
\hline \multicolumn{4}{|c|}{ Number of coronary arteries narrowed } \\
\hline 1 & $175(76 \%)$ & $45(43 \%)$ & $<0.001$ \\
\hline$>1$ & $55(24 \%)$ & $60(57 \%)$ & $<0.001$ \\
\hline \multicolumn{4}{|l|}{ PCI characteristics } \\
\hline$\geq 2$ stent implantations & $39(19 \%)$ & $29(44 \%)$ & $<0.001$ \\
\hline Stent implantation & $100 \%$ & $100 \%$ & 1.000 \\
\hline Bare metal stent & $176(87 \%)$ & $59(89 \%)$ & 0.783 \\
\hline Drug-eluting stent & $26(13 \%)$ & $7(11 \%)$ & \\
\hline Stent length, $\mathrm{mm}$ & $20.5 \pm 4.9$ & $19.2 \pm 5.7$ & 0.145 \\
\hline Stent diameter, $\mathrm{mm}$ & $3.26 \pm 0.49$ & $3.17 \pm 0.41$ & 0.252 \\
\hline Follow-up, months & $18 \pm 10$ & $18 \pm 12$ & 0.590 \\
\hline All-cause mortality & $41(18 \%)$ & $33(31 \%)$ & 0.005 \\
\hline
\end{tabular}

Data are expressed as mean \pm SD for normally distributed data and percentage $(\%)$ for categorical variables. SBP = Systolic blood pressure; $\mathrm{DBP}=$ diastolic blood pressure; $\mathrm{BMI}=$ body mass index; $\mathrm{LVEF}=$ left ventricular ejection fraction; $\mathrm{PCI}=$ percutaneous coronary intervention; $\mathrm{CABG}=$ coronary artery bypass graft.

statistically significant $(\mathrm{p}=0.009)$. There were no significant differences in the presence of hypertension, diabetes mellitus and smoking status between groups. LVEF was significantly higher in the low SYNTAX group than in the high SYNTAX group (54 \pm 9 vs. $51 \pm 9, \mathrm{p}=0.004$ ). Percutaneous coronary intervention was the preferred treatment option in the low SYNTAX group while coronary artery bypass graft was preferred in the high SYNTAX group ( $\mathrm{p}<0.001$ for each). A greater proportion of patients had multivessel disease (more than $50 \%$ occlusion for multiple coronary arteries) in the high SYNTAX group. In all patients, stent implantation was performed. Implanted stent types were similar between the groups $(\mathrm{p}=0.783)$. In respect of stent properties, there was no
Table 2. Laboratory parameters of patients

\begin{tabular}{lccr}
\hline & $\begin{array}{c}\text { Low SYNTAX } \\
\text { group }(\mathrm{n}=230)\end{array}$ & $\begin{array}{c}\text { High SYNTAX } \\
\text { group }(\mathrm{n}=105)\end{array}$ & $\begin{array}{l}\mathrm{p} \\
\text { value }\end{array}$ \\
\hline Hemoglobin, g/l & $13.8 \pm 2.1$ & $13.6 \pm 2.3$ & 0.467 \\
Platelets, $/ \mathrm{mm}^{3}$ & $274.4 \pm 88.0$ & $256.5 \pm 75.4$ & 0.071 \\
WBC, $10^{3} / \mu \mathrm{l}$ & $9.61 \pm 3.25$ & $9.70 \pm 3.41$ & 0.835 \\
Neutrophils, \% & $67.7 \pm 12.0$ & $69.8 \pm 11.8$ & 0.135 \\
Lymphocytes, \% & $22.3 \pm 10.7$ & $19.9 \pm 9.7$ & 0.064 \\
Neutrophil/lymphocyte ratio & $4.7 \pm 3.0$ & $5.8 \pm 3.6$ & 0.098 \\
RDW, \% & $14.2 \pm 1.2$ & $15.2 \pm 1.8$ & $<0.001$ \\
MPV, fl & $9.0 \pm 0.9$ & $9.1 \pm 1.1$ & 0.609 \\
Glucose, mg/dl & $133.6 \pm 46.3$ & $134.4 \pm 47.6$ & 0.885 \\
Creatinine, mg/dl & $0.9 \pm 0.2$ & $1.1 \pm 0.3$ & $<0.001$ \\
LDL-C, mg/dl & $108.6 \pm 20.7$ & $108.9 \pm 21.5$ & 0.901 \\
HDL-C, mg/dl & $38.7 \pm 8.5$ & $37.2 \pm 8.7$ & 0.164 \\
Total cholesterol, mg/dl & $170.4 \pm 38.1$ & $187.2 \pm 41.8$ & 0.001 \\
Triglycerides, mg/dl & $128.9 \pm 57.2$ & $149.6 \pm 66.3$ & 0.006 \\
hs-CRP, mg/l & $19.2 \pm 13.0$ & $27.1 \pm 19.3$ & 0.004 \\
Troponin-I, ng/ml & $3.4 \pm 1.1$ & $2.9 \pm 1.0$ & 0.353 \\
CK-MB, U/l & $51.2 \pm 25.5$ & $54.9 \pm 32.0$ & 0.573 \\
\hline
\end{tabular}

Data are expressed as mean \pm SD or median for normally distributed data and percentage (\%) for categorical variables. SS = Syntax score; $\mathrm{WBC}=$ white blood cell; $\mathrm{RDW}=$ red cell distribution width; MPV = mean platelet volume; LDL-C = low density lipoprotein cholesterol; HDL-C = high density lipoprotein cholesterol; Hs-CRP = high sensitivity C-reactive protein; $\mathrm{CK}-\mathrm{MB}=$ peak creatine kinase-MB. Lymphocyte, Hs-CRP and CK-MB are expressed as mean \pm median for normally distributed data and percentage (\%) for categorical variables.

significant difference in stent length $(20.5 \pm 4.9$ vs. $19.2 \pm$ $5.7 \mathrm{~mm}, \mathrm{p}=0.145)$ or stent diameter $(3.26 \pm 0.49$ vs. $3.17 \pm 0.41 \mathrm{~mm}, \mathrm{p}=0.252$ ) between the groups.

The RDW levels of patients were significantly higher in the high SYNTAX group (15.2 \pm 1.8 vs. $14.2 \pm 1.2$, p < 0.001 ; table 2). With respect to other laboratory analyses, creatinine, total cholesterol and triglycerides were significantly higher in the high SXscore group ( $<<0.001, \mathrm{p}=$ 0.001 and $p=0.006$, respectively). hs-CRP was also significantly higher in the high SXscore group than in the low SXscore group $(27.1 \pm 19.3$ vs. $19.2 \pm 13.0, \mathrm{p}=0.004)$.

There was a significant and positive correlation between RDW levels and SXscore $(r=0.460, \mathrm{p}<0.001)$. There was also a significant, but not strong, correlation between RDW levels and hs-CRP $(r=0.180, p=0.001)$.

Some variables associated with a high SXscore were significantly different between the groups. On multivariate linear regression analyses, the RDW levels $(\beta=0.969$, $\mathrm{p}<0.001)$, LDL-C $(\beta=0.059, \mathrm{p}=0.003)$ and troponin-I $(\beta=0.170, p=0.014)$ remained independent correlates of a high SXscore in patients with NSTEMI (table 3). The 
Table 3. Effects of various variables on high SXscores in multivariate linear regression analyses

\begin{tabular}{lllr}
\hline Variables & $\beta$ & SE & p value \\
\hline Age & 0.049 & 0.029 & 0.092 \\
RDW & 0.969 & 0.231 & $<0.001$ \\
Total cholesterol & 0.020 & 0.011 & 0.076 \\
LDL-C & 0.059 & 0.019 & 0.003 \\
Hs-CRP & 0.023 & 0.015 & 0.110 \\
Troponin-I & 0.170 & 0.069 & 0.014 \\
\hline
\end{tabular}

Variables adjusted for age, LDL-C, hs-CRP, and troponin-I.

Table 4. Multivariate Cox regression analysis of patients for allcause mortality

\begin{tabular}{llc}
\hline Risk factors & HR $(95 \% \mathrm{CI})$ & p value \\
\hline SXscore & $1.04(1.00-1.08)$ & 0.019 \\
Age & $1.04(1.01-1.06)$ & 0.003 \\
LVEF & $0.95(0.92-0.98)$ & $<0.001$ \\
RDW & $0.97(0.83-1.12)$ & 0.694 \\
\hline
\end{tabular}

ROC curve of RDW levels on admission was used for predicting a high SXscore. An RDW level $>14.25 \%$ measured on admission had a $66 \%$ sensitivity and $64 \%$ specificity in predicting a high SXscore.

The independent predictors of all-cause mortality in the study population were demonstrated with Cox regression analysis. SXscore (hazard ratio, HR 1.04, 95\% CI $1.00-1.08, \mathrm{p}=0.019$ ), age (HR 1.04, 95\% CI 1.01-1.06, $\mathrm{p}=0.003$ ) and LVEF (HR 0.95, 95\% CI 0.92-0.98, p < 0.001 ) were independent predictors of all-cause mortality in patients with NSTEMI (table 4).

The median follow-up period was 18 months (1-38 months).The all-cause mortality rate was higher in the high SXscore group than in the low SXscore group (27 vs. $19 \%, \mathrm{p}=0.005$; table 1). In the survival analysis, however, the all-cause mortality rate was not significantly different between the high and the low RDW group (log-rank, $\mathrm{p}=$ 0.621 ; fig. 1).

\section{Discussion}

We showed a positive association between high levels of RDW and the severity of coronary artery disease in patients with NSTEMI. We also demonstrated that high lev-

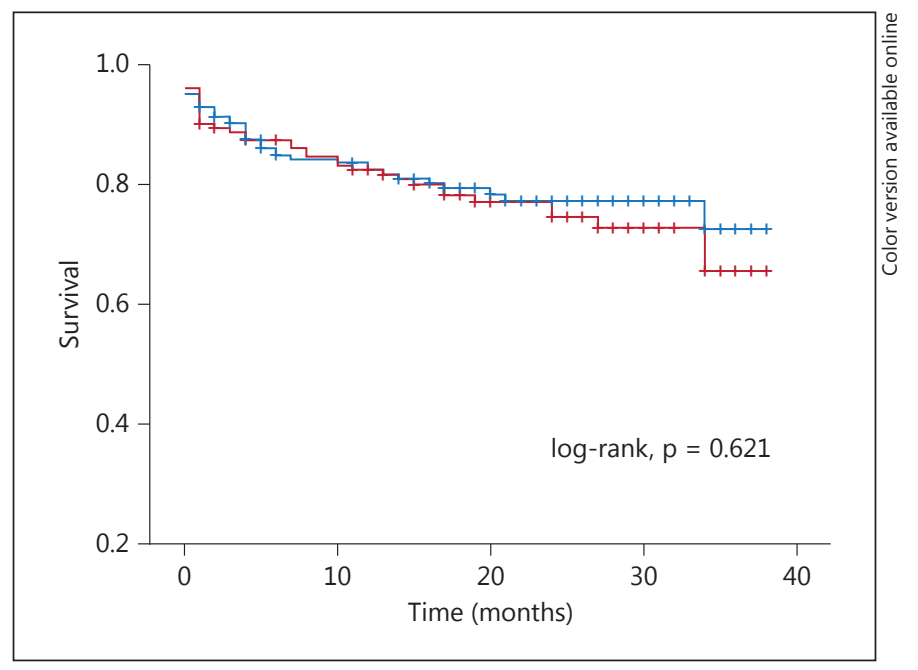

Fig. 1. Kaplan-Meier survival estimates by RDW.

els of RDW on admission correlated with a high SXscore. Although age, troponin level and LVEF predicted allcause mortality, no association was found between RDW levels and all-cause mortality.

The RDW as a marker of the variability in erythrocyte volume is a routinely available component of the complete blood count. In patients with ineffective red cell production (such as iron deficiency, B12 or folate deficiency and hemoglobinopathies), increased red cell destruction (such as hemolysis) and blood transfusion, the RDW levels can be elevated [11]. The RDW reflects variability in the size of circulating red cells (anisocytosis) and is routinely reported by analyzers as part of routine complete blood counts. Several studies in the literature have reported that elevated RDW levels are significantly associated with a poor prognosis in the setting of heart failure [12], acute myocardial infarction [4] and stable angina pectoris [5]. RDW is now recognized as a global marker of chronic inflammation and oxidative stress [13]. However, the underlying biological mechanisms remain unclear. In our study, we demonstrated a statistically significant correlation between RDW and hs-CRP.

We showed a positive association between high levels of RDW and the severity of coronary artery disease in patients with NSTEMI. We also demonstrated that high levels of RDW on admission correlated with a high SXscore. The underlying pathophysiological mechanism of coronary artery disease is the atherosclerotic process $[14,15]$. Atherosclerosis is a multifactorial disease process that is affected by multiple pathophysiological factors. One of 
these factors is inflammation [16-18]. Inflammation has a key role in the atherosclerotic process $[19,20]$. In a recent study, Lippi et al. [21] demonstrated a relationship between generalized inflammation and elevated levels of RDW. They demonstrated an association of RDW with hs-CRP, which is an indicator of inflammation. This relationship between high RDW levels and generalized inflammation, particularly that associated with CRP levels, is yet to be clarified. The most plausible hypothesis is that higher RDW levels are associated with increased inflammation. The increased inflammation might be causing the immature erythrocytes in the bone marrow to enter the circulation, thus leading to anisocytosis (and increased RDW levels). Inflammatory cytokines, which are activated in acute conditions of coronary artery disease such as STEMI and NSTEMI, have been found to suppress erythrocyte maturation. Due to this situation, juvenile erythrocytes enter into the circulation [22].

In this study, for the first time, we demonstrated that the RDW levels on admission were independently associated and correlated with a high SXscore in NSTEMI patients. The SXscore is an anatomic scoring system and quantifies the properties of a lesion, including complexity, morphology and location in the coronary tree. High SXscores predict adverse outcomes after percutaneous coronary intervention in patients with coronary artery disease who underwent revascularization [23, 24]. Recently, two studies demonstrated that RDW was independently associated with the presence and coronary complexity of coronary artery disease in patients with STEMI and stable angina pectoris as assessed by the SXscore [4, 5]. It was demonstrated that a higher SXscore predicts worse short- and long-term clinical outcomes [25].

Several studies have evaluated the relation between increased RDW and morbidity and mortality of coronary artery disease. Recent studies showed that an increased RDW was independently associated with in-hospital and long-term all-cause mortality and coronary events in STEMI and NSTEMI patients and in an unselected population of male patients referred for coronary angiography $[3,26,27]$. Also, a high level of RDW on admission was independently associated with worse reperfusion and increased risk of in-hospital and long-term cardiovascular mortality in patients with STEMI [27]. Unlike in these studies, no association was found between RDW levels and all-cause mortality in our study. In contrast to RDW levels, we found a significant association between high SXscore and all-cause mortality in our study. As stated, in contrast to the recent literature, no association was found in our study between RDW levels and all-cause mortality.
This may be due to a number of reasons. Our study was represented by a small number of patients and a singlecenter experience. Further, our study design was crosssectional. Therefore, further prospective multicenter studies with a larger sample size should be planned in order to evaluate the association of RDW with mortality. Another limitation of our study is that we only measured hemoglobin levels, but not other factors such as iron, vitamin B12 and folate. A further limitation is that we only assessed hs-CRP levels, although we speculate that inflammation may be an actual pathophysiological mechanism between RDW levels and the severity of coronary artery disease. In addition, RDW was assessed only once. We have no data on changes in RDW levels during the course of hospital stay.

\section{Conclusion}

RDW levels were independently associated with a high SXscore but were not associated with long-term mortality in patients with NSTEMI.

\section{Disclosure Statement}

The authors have no conflicts of interest to declare.

\section{References}

1 Dabbah S, Hammerman H, Markiewicz W, et al: Relation between red cell distribution width and clinical outcomes after acute myocardial infarction. Am J Cardiol 2010;105: 312-317.

2 Sangoi MB, Da Silva SH, da Silva JE, et al: Relation between red blood cell distribution width and mortality after acute myocardial infarction. Int J Cardiol 2011;146:278-280.

3 Azab B, Torbey E, Hatoum H, et al: Usefulness of red cell distribution width in predicting all-cause long-term mortality after nonST-elevation myocardial infarction. Cardiology 2011;119:72-80.

4 Isik T, Uyarel H, Tanboga IH, et al: Relation of red cell distribution width with the presence, severity, and complexity of coronary artery disease. Coron Artery Dis 2012;23:51-56.

5 Akin F, Köse N, Ayça B, et al: Relation between red cell distribution width and severity of coronary artery disease in patients with acute myocardial infarction. Angiology 2013; 64:592-596.

6 Osadnik T, Strzelczyk J, Hawranek M, et al: Red cell distribution width is associated with long-term prognosis in patients with stable coronary artery disease. BMC Cardiovasc Disord 2013;13:113.
Sahin/Akpek/Sarli/Baktir/Savas / Karadavut/Elcik/Saglam/Kaya/Arinc 
7 Drüeke TB: Anemia treatment in patients with chronic kidney disease. N Engl J Med 2013;368:387-389.

8 Leaman DM, Brower RW, Meester GT, et al: Coronary artery atherosclerosis: severity of the disease, severity of angina pectoris and compromised left ventricular function. Circulation 1981;63:285-299.

9 Sianos G, Morel MA, Kappetein AP, et al: The SYNTAX Score: an angiographic tool grading the complexity of coronary artery disease. EuroIntervention 2005;1:219-227.

10 Akpek M, Kaya MG, Uyarel H, et al: The association of serum uric acid levels on coronary flow in patients with STEMI undergoing primary PCI. Atherosclerosis 2011;219:334341.

11 Förhécz Z, Gombos T, Borgulya G, et al: Red cell distribution width in heart failure: prediction of clinical events and relationship with markers of ineffective erythropoiesis, inflammation, renal function, and nutritional state. Am Heart J 2009;158:659-666.

12 Felker GM, Allen LA, Pocock SJ, et al CHARM Investigators: Red cell distribution width as a novel prognostic marker in heart failure: data from the CHARM Program and the Duke Databank. J Am Coll Cardiol 2007; 50:40-47.

13 Tsuboi S, Miyauchi K, Kasai T, et al: Impact of red blood cell distribution width on longterm mortality in diabetic patients after per- cutaneous coronary intervention. Circ J 2013; 77:456-461.

14 Kaya MG, Uyarel H, Akpek M, et al: Prognostic value of uric acid in patients with ST-elevated myocardial infarction undergoing primary coronary intervention. Am J Cardiol 2012;109:486-491.

15 Sahin O, Akpek M, Elcik D, et al: Bilirubin levels and the burden of coronary atherosclerosis in patients with STEMI. Angiology 2013; 64:200-204.

16 Libby P, Ridker PM, Maseri A: Inflammation and atherosclerosis. Circulation 2002;105: 1135-1143.

17 Walia M, Kwan CY, Grover AK: Effects of free radicals on coronary artery. Med Princ Pract 2003;12:1-9.

18 Hassouna HI: Thrombophilia and hypercoagulability. Med Princ Pract 2009;18:429-440.

19 Libby P: What have we learned about the biology of atherosclerosis? The role of inflammation. Am J Cardiol 2001;88:3J-6J.

20 Ayhan SS, Oztürk S, Erdem A, et al: Relation of neutrophil/lymphocyte ratio with the presence and severity of coronary artery ectasia (in Turkish). Turk Kardiyol Dern Ars 2013; 41:185-190.

21 Lippi G, Targher G, Montagnana M, et al: Relation between red blood cell distribution width and inflammatory biomarkers in a large cohort of unselected outpatients. Arch Pathol Lab Med 2009;133:628-632.
22 Pierce CN, Larson DF: Inflammatory cytokine inhibition of erythropoiesis in patients implanted with a mechanical circulatory assist device. Perfusion 2005;20:83-90.

23 Serruys PW, Morice MC, Kappetein AP, et al; SYNTAX Investigators: Percutaneous coronary intervention versus coronary artery bypass grafting for severe coronary artery disease. N Engl J Med 2009;360:961-972.

24 van Gaal WJ, Ponnuthurai FA, Selvanayagam J, et al: The SYNTAX score predicts peri-procedural myocardial necrosis during percutaneous coronary intervention. Int J Cardiol 2009; 135:60-65.

25 Uyarel H, Ergelen M, Cicek G, et al: Red cell distribution width as a novel prognostic marker in patients undergoing primary angioplasty for acute myocardial infarction. Coron Artery Dis 2011;22:138-144.

26 Cavusoglu E, Chopra V, Gupta A, et al: Relation between red blood cell distribution width (RDW) and all-cause mortality at two years in an unselected population referred for coronary angiography. Int J Cardiol 2010;141: 141-146.

27 Karabulut A, Uyarel H, Uzunlar B, et al: Elevated red cell distribution width level predicts worse postinterventional thrombolysis in myocardial infarction flow reflecting abnormal reperfusion in acute myocardial infarction treated with a primary coronary intervention. Coron Artery Dis 2012;23:68-72. 